\title{
PERFECT MAPPINGS AND CERTAIN INTERIOR IMAGES OF M-SPACES(1)
}

\author{
BY
}

\author{
J. M. WORRELL, JR. AND H. H. WICKE
}

ABSTRACT. The main theorems of this paper show that certain conditions (called $\lambda_{c}, \lambda_{b}, \beta_{c}$, and $\beta_{b}$ ) are invariant, in the presence of $T_{0}$-regularity, under the application of closed continuous peripherally compact mappings. Interest in these conditions lies in the fact that they may be used to characterize certain regular $T_{0}$ open continuous images of some classes of $M$-spaces in the sense of $\mathrm{K}$. Morita, and in the fact that they are preserved by open continuous mappings with certain appropriate additional conditions. For example, the authors have shown that a regular $T_{0}$-space is an open continuous image of a paracompact Čech complete space if and only if the space satisfies condition $\lambda_{b}$ [Pacific J. Math. 37 (1971), 265-275]. Moreover, in the same paper it is shown that if a completely regular $T_{0}$-space satisfies condition $\lambda_{b}$ then any $T_{0}$ completely regular open continuous image of it also satisfies $\lambda_{b}$. These results together with the results of the present paper and certain known results lead to the following theorem: The smallest subclass of the class of regular $T_{0}$-spaces which contains all paracompact Čech complete spaces and which is closed with respect both to the application of perfect mappings and to the application of open continuous mappings preserving $T_{0}$-regularity is the subclass satisfying condition $\lambda_{b}$. Similar results are obtained for the regular $T_{0}$-spaces satisfying $\lambda_{c}, \beta_{b}$, and $\beta_{c}$. The other classes of $M$-spaces involved are the regular $T_{0}$ complete $M$-spaces (i.e., spaces which are quasi-perfect preimages of complete metric spaces), $T_{2}$ paracompact $M$-spaces, and regular $T_{0} M$-spaces. In the last two cases besides the interiority of the mappings the notion of uniform $\lambda$-completeness, which generalizes compactness of a mapping, enters. (For details see General Topology and Appl. 1 (1971), 85-100.)

The proofs are accomplished through the use of two basic lemmas on closed continuous mappings satisfying certain additional conditions.

1. Introduction. The main theorems of this paper display some invariants of perfect mappings and certain other closed continuous mappings having regular $T_{0}$ domains. The invariants are given in the form of certain sequence conditions $\lambda_{c}$,

Presented to the Society, August 28, 1970; received by the editors January 11, 1971. AMS (MOS) subject classifications (1970). Primary 54C10, 54E99; Secondary 54C99, 54D20.

Key words and phrases. Čech complete spaces, $\beta_{b}$-space, $\beta_{c}$-space, $\lambda_{b}$-space, $\lambda_{c}$ space, $\mu$-space, complete $i l$-space, $q$-space, point-countable covering, first countable-like closed continuous mappings, primitive sequence, monotonically contracting sequence, (absolute) set of interior condensation, perfect mappings, open continuous mappings.

(1) This work was supported by the United States Atomic Energy Commission. 
$\lambda_{b}, \beta_{c}, \beta_{b}$. These were introduced in [15], where examples and discussion may be found.

Some reasons for interest in these conditions may be elicited from the following results: I. A regular $T_{0}$-space is an open continuous image of a paracompact Čech complete space (regular $T_{0}$ complete $M$-space $\left({ }^{2}\right)$ ) if and only if it satisfies condition $\lambda_{b}\left(\lambda_{c}\right)$. II. A regular $T_{0}$-space is an open continuous uniformly $\lambda$-complete( $\left.{ }^{3}\right)$ image of a paracompact $M$-space (regular $T_{0} M$-space) if and only if it satisfies $\beta_{b}\left(\beta_{c}\right)$. (The sufficiency part of these theorems is proved in [15]. The proof of necessity is to be submitted.)

Consider the first parts of I and II. Frolik [4] showed that perfect mappings between Tychonoff spaces preserve Čech completeness and Michael [9] showed that closed continuous mappings preserve $T_{2}$ paracompactness. Filippov [2] and Ishii [6] showed that perfect mappings preserve the property of being a $T_{2}$ paracompact $M$-space (equivalently, a $T_{2}$ paracompact $p$-space). However from [14] it follows that there exists an open continuous uniformly $\lambda$-complete mapping of a complete metric space onto a space which is neither paracompact nor an M-space. With regard to the second parts of I and II, Morita gave an example [13] of a perfect mapping of a Hausdorff locally compact $M$-space onto a space which is not an $M$-space. Contrastingly, Theorem 2.1 may be applied with the propositions of I and II to obtain the following results.

Theorem 1.1. The smallest subclass of the class of regular $T_{0}$-spaces which contains all paracompact $\breve{C} e c h$ complete spaces (respectively, all regular $T_{0}$ complete M-spaces) and which is closed with respect both to the application of perfect mappings and to the application of open continuous mappings preserving $T_{0}$ regularity is the subclass satisfying condition $\lambda_{b}$ (respectively, condition $\lambda_{c}$ ).

Theorem 1.2. The smallest subclass of the class of regular $T_{0}$ spaces which contains all paracompact Hausdorff p-spaces (respectively, all regular $T_{0}-M$ spaces) and which is closed with respect to both the application of perfect mappings and to the application of uniformly $\lambda$-complete open continuous mappings preserving $T_{0}$-regularity is the subclass satisfying condition $\beta_{b}$ (respectively, condition $\beta_{c}$ ).

Another point of interest arises from Worrell's example [22] of a $p$-space which is mapped onto a space which is not a $p$-space by a perfect mapping. The authors have introduced and elaborated the concept of a $\mu$-space [17] in which the finite intersection property of the $p$-space concept is replaced with a decreasing sequence.

(2) A topological space is said to be a complete $M$-space if and only if it is a quasiperfect pre-image of a complete metric space. The term $M$-space is used in the sense of Morita [12].

(3) Uniformly $\lambda$-complete mappings are defined in [15]. 
Below it is shown that a completely regular $T_{0}$-space is a $\mu$-space (complete $\mu$ space) if and only if it satisfies $\beta_{b}\left(\lambda_{b}\right)$. Thus the result that such spaces are preserved by perfect mappings with completely regular $T_{0}$ domains shows a significant difference between the concept of a $\mu$-space and that of a $p$-space.

Two final remarks of a general nature remain to be made. The general topic of this paper concerns principles of uniformization for topological spaces. Examination of the definitions below shows that the sequence conditions given are variations of a fundamental uniformization principle in the first countable case: the property of being an essentially $T_{1}$ space having a base of countable order. This is clear on recalling that a space has this property if and only if there exists a monotonically contracting sequence $\left(\biguplus_{n}\right)$ of collections of open sets covering the space such that if $\left(G_{n}\right)$ is a sequence such that each $G_{n+1} \subset G_{n}$, each $G_{n} \in \varrho_{n}$, and $x \in \bigcap\left\{G_{k}: k \in N\right\}$, then $\left\{G_{k}: k \in N\right\}$ is a base at $x$ [19]. This helps account for a close relationship in method of the present paper to methods of previous papers treating bases of countable order and developable spaces (cf. especially [19], [20], [21]) and also for the essential unity of treatment of the proofs of of the main results. The present article shows the satisfactory behavior of the principles of uniformization $\lambda_{c}, \lambda_{b}, \beta_{c}, \beta_{b}$ under perfect mappings. Our final remark is to call attention to the important role which compactness in the sense of Fréchet [3] plays in the concepts and in the proof sof the principal results.

Notation and terminology. The notation and terminology used here conforms closely with that of Kelley [7]. If $A$ is a set, $\mathrm{Fr}_{\mathrm{r}}(A)$ denotes the boundary of $A$ and Int $(A)$ denotes the interior of $A$. The letter $N$ denotes the set of positive integers, and letters $i, j, k$, and $n$ are used to signify members of $N$. The notation $\left(U_{n}\right)_{n \in N}$ is used for a sequence, i.e., a function $U$ with domain $N$; this notation will be abbreviated to $\left(U_{n}\right)$. A sequence $\left(U_{n}\right)$ of sets is said to be decreasing if and only if, for each $n, U_{n+1} \subset U_{n}$. A representative of a sequence $\left(\mathbb{P}_{n}\right)$ of sets is, by definition, a sequence $\left(B_{n}\right)$ such that, for each $n, B_{n} \in \mathbb{P}_{n}$. A mapping is said to be peripherally compact if and only if the inverse image of every point has a compact boundary. A mapping is said to be quasi-perfect [13] (perfect) if and only if it is a closed continuous mapping such that the inverse image of every point is countably compact (compact). If $\mathbb{P}$ is a collection of sets $U \mathbb{Q}$ denotes the union of the collection. An expression such as " $\bigcup \mathcal{F} \cup M$ " denotes the union of $\bigcup \mathfrak{F}$ and $M$.

2. Definitions and results. The definitions of the basic concepts and the statements of the main theorems are given here. A discussion of the conditions of Definitions 2.2 and 2.3 together with examples is given in [15].

Definition 2.1 [18]. Let $M \subset X$. A sequence $\left(\mathcal{C}_{n}\right)$ of collections of subsets of $X$ covering $M$ is said to be a monotonically contracting sequence of $M$ in $X$ if and only if, for each $n \in N$, if $x \in A \in \mathcal{C}_{n}$ and $x \in M$, there exists $B \in \mathcal{C}_{n+1}$ such 
that $x \in B \subset A$. If $M=X$, the sequence is called monotonically contracting.

Definition 2.2 [15]. A topological space $X$ is said to satisfy condition $\lambda_{c}\left(\lambda_{b}\right)$ if and only if there exists a monotonically contracting sequence $\left(\biguplus_{n}\right)$ such that: (1) Each $\varrho_{n}$ is a collection of open subsets of $X$ covering $X$. (2) If $\left(G_{n}\right)$ is a decreasing representative of $\left(\mathcal{G}_{n}\right)$ and each $G_{n}$ is nonempty then $B=\bigcap\left\{\bar{G}_{n}: n \in N\right\}$ is nonempty and countably compact (compact) and $U$ open and $B \subset U$ implies that some $G_{k} \subset U$.

Definition 2.3 [15]. A topological space $X$ is said to satisfy condition $\beta_{c}\left(\beta_{b}\right)$ if and only if there exists a monotonically contracting sequence $\left(\mathcal{G}_{n}\right)$ such that: (1) Each $\varrho_{n}$ is a collection of open subsets of $X$ covering $X$. (2) If $\left(G_{n}\right)$ is a decreasing representative of $\left(\varrho_{n}\right)$ then if $B=\bigcap\left\{\bar{G}_{n}: n \in N\right\}$ is nonempty it is countably compact (compact) and $U$ open and $B \subset U$ implies that some $G_{k} \subset U$.

Theorem 2.1. Suppose $X$ is a regular $T_{0}$-space and there is a peripherally compact closed continuous mapping of $X$ onto $Y$. If $X$ satisfies any of the conditions $\lambda_{c}, \lambda_{b}, \beta_{c}$, or $\beta_{b}$ then $Y$ satisfies the same condition.

Theorem 2.2. Suppose $f$ is a closed continuous mapping of a regular $T_{0}$-space $X$ onto a space $Y$ such that if $U$ is open in $Y$ and $y \in U$ there is a sequence $\left(V_{n}\right)$ of open sets containing $y$ such that any representative of $\left(V_{n}\right)$ bas a cluster point in U.(4) Also suppose that for each $y \in Y$, if $\mathcal{H}$ is a collection of open subsets of $X$ covering $\operatorname{Fr}\left(f^{-1}(y)\right)$, then there exists a point-countable collection of open sets covering $\operatorname{Fr}\left(f^{-1}(y)\right)$ and refining $\mathcal{H}$. Then if $X$ satisfies any of the conditions $\lambda_{c}, \lambda_{b}, \beta_{c}$, or $\beta_{b}$ then $Y$ satisfies the same condition.

The concepts defined just below have been introduced and elaborated in [17]. The class of $\mu$-spaces includes the class of $p$-spaces of Arhangel'skii [1], and the class of complete $\mu$-spaces includes the class of Čech complete spaces. The introduction of these concepts permits a more satisfactory theory of invariance under peripherally compact closed continuous mappings and certain open continuous mappings as is shown by Theorem 2.3 and the results of [15], respectively.

Definition 2.4 [17]. A $T_{1}$-space $X$ is called a $\mu$-space if and only if there exists a sequence $\left(\biguplus_{n}\right)$ of collections of open subsets of the Wallman compactification $\omega X$ of $X$ such that: (1) $\left(\biguplus_{n}\right)$ is a monotonically contracting sequence of $X$ in $\omega X$. (2) If $\left(G_{n}\right)$ is a decreasing representative of $\left(\biguplus_{n}\right)$, then if $\cap\left\{G_{n}: n \in N\right\}$ contains a point of $X$ it is a subset of $X$.

Remark 2.1. If $X$ is a completely regular $T_{0}$-space, then $X$ is a $\mu$-space if and only if there is a monotonically contracting sequence $\left(\mathcal{G}_{n}\right)$ of $X$ in $b X$ of collections of open subsets of some $T_{2}$ compactification $b X$ of $X$ having property (2). In this case if there is such a sequence in some $T_{2}$ compactification, there is one in every $T_{2}$ compactification.

(4) I.e., $Y$ has a base each element of which is a $q$-space in the sense of [10]. 
Remark 2.2. Lemma 3.6 gives an intrinsic characterization of completely regular $T_{0} \mu$-spaces.

Definition 2.5 [18]. A subset $M$ of a topological space $X$ is said to be a set of interior condensation in $X$ if and only if there exists a monotonically contracting sequence $\left(\varrho_{n}\right)$ of $M$ in $X$ of collections of open subsets of $X$ covering $M$ such that if $\left(G_{n}\right)$ is a decreasing representative of $\left(\biguplus_{n}\right)$ then $\bigcap\left\{G_{n}: n \in N\right\} \subset M$.

Definition 2.6. A completely regular $T_{0}$-space $X$ is called a complete $\mu$-space if and only if it is a set of interior condensation in its Stone-Čech compactification.

Remark 2.3. A complete $\mu$-space is a set of interior condensation in any its $T_{2}$ compactifications (indeed, in any Hausdorff space in which it can be densely embedded). This justifies the alternative terminology of set of absolute interior condensation and shows that this generalizes the idea of Čech completeness.

Remark 2.4. Lemma 3.6 gives an intrinsic characterization of complete $\mu$ spaces. For another characterization and further information see [18].

Theorem 2.3. Suppose $X$ and $Y$ are completely regular $T_{0}$-spaces and there is a peripherally compact closed continuous mapping of $X$ onto $Y$. Then if $X$ is a $\mu$-space (respectively, a complete $\mu$-space) so is $Y$.

Theorem 2.4. Suppose $X$ and $Y$ are completely regular $T_{0}$-spaces and $f$ is a closed continuous mapping of $X$ onto $Y$. If $f$ and $Y$ bave the properties stated in the bypothes is of Theorem 2.2, then if $X$ is a $\mu$-space (respectively, a complete $\mu$-space) so is $Y$.

3. Lemmas on closed mappings; other results. The first two lemmas below state equivalent forms of the basic conditions in the regular $T_{0}$ case which are convenient for use in the proofs. The next three lemmas are useful in several proofs. Lemma 3.6 characterizes completely regular $T_{0} \mu$-spaces and complete $\mu$-spaces in terms of conditions $\beta_{b}$ and $\lambda_{b}$ respectively. Lemma 3.7 is a fundamental lemma on closed mappings. The key idea of the proof is found in the proof of the Lemma of [21]. Lemma 3.8 is the main lemma used in proving the theorems. It is similar to other lemmas used in first countable cases in [20], [21].

Lemma 3.1 [15]. Suppose $X$ is a regular $T_{0}$-space. Then in $X$ the condition $\lambda_{c}\left(\lambda_{b}\right)$ is equivalent to: There exists a sequence $\left(\biguplus_{n}\right)$ of collections of open sets covering $X$ such that: (1) For each $n \in N$, if $x \in G \in \mathcal{G}_{n}$ there exists $G^{\prime} \in \mathcal{G}_{n+1}$ sucb that $x \in G^{\prime}$ and $\bar{G}^{\prime} \subset G$. (2) If $\left(G_{n}\right)$ is a sequence sucb that $\varnothing \neq \bar{G}_{n+1} \subset G_{n}$ $\epsilon \mathcal{G}_{n}$ for all $n \in N$, then $B=\bigcap\left\{G_{n}: n \in N\right\}$ is nonempty and countably compact (compact) and every open $U$ which includes $B$ also includes some $G_{n}$.

Lemma 3.2 [15]. If $X$ is a regular $T_{0}$-space the condition $\beta_{c}\left(\beta_{b}\right)$ is equivalent to the condition obtained from that of Lemma 3.1 by replacing (2) with: 
$\left(2^{\prime}\right)$ If $\left(G_{n}\right)$ is a sequence such that $\bar{G}_{n+1} \subset G_{n} \in \mathcal{G}_{n}$ for all $n \in N$, then $B=$ $\bigcap\left\{G_{n}: n \in N\right\}$ is countably compact (compact) and if $B$ is nonempty every open $U$ which includes $B$ also includes some $G_{n}$.

Definition 3.1. If $S$ is a subset of a set $X$ a sequence $\left(\mathcal{H}_{n}\right)$ is said to be a primitive sequence [16] of $S$ in $X$ if and only if for each $n \in N$ these conditions are satisfied: (1) $\mathcal{H}_{n}$ is a well-ordered collection of subsets of $X$ covering $S$. (2) If $H \in H_{n}$ there exists $x \in H \cap S$ not in any predecessor of $H$. (3) If $x \in S, n<k$, and $H$ and $H^{\prime}$ are the first elements of $\mathcal{H}_{n}$ and $\mathcal{H}_{k}$, respectively, that contain $x$, then $H \supset H^{\prime}$.

Lemma 3.3 [16]. If $S$ is a subset of a set $X$ and $\left(\biguplus_{n}\right)$ is a monotonically contracting sequence of $S$ in $X$, there exists a primitive sequence $\left(\mathcal{H}_{n}\right)$ of $S$ in $X$ such that each $\mathcal{H}_{n} \subset \mathfrak{S}_{n}$.

The following lemma formalizes an argument used repeatedly in a number of publications of the authors.

Lemma 3.4. Suppose $\left(\mathcal{H}_{n}\right)$ is a primitive sequence of $S$ in $X$ and $\left(G_{n}\right)$ is a decreasing sequence of sets such that for each $n$ there exists $y_{n} \in G_{n} \cap S$ such that the first element of $\mathcal{H}_{n}$ that contains $y_{n}$ includes $G_{n}$. Then there exists a decreasing representative $\left(H_{n}\right)$ of $\left(\mathcal{H}_{n}\right)$ such that, for each $n, H_{n}$ is the first element of $\mathcal{H}_{n}$ that includes a term of $\left(G_{n}\right)$.

Proof. For each $n$ there exists a first $H_{n} \in \mathcal{H}_{n}$ that includes a term of $\left(G_{n}\right)$. For each $n$ there exists $j>n+1$ such that $G_{j} \subset H_{n} \cap H_{n+1}$. Let $H$ denote the first element of $\mathcal{H}_{n}$ that contains $y_{j}$. Since $\left(\mathcal{H}_{n}\right)$ is a primitive sequence, $H$ includes the first element $H^{\prime}$ of $\mathcal{H}_{j}$ that contains $y_{j}$. Thus $H \supset H^{\prime} \supset G_{j}$. Therefore $H$ does not precede $H_{n}$. Since $y_{j} \in H_{n}$ it follows that $H=H_{n}$. Similarly $H_{n+1}$ is the first element of $\mathcal{H}_{n+1}$ that contains $y_{j}$. Therefore $H_{n+1} \mathrm{CH}_{n}$.

Definition 3.2 and Lemma 3.5 are introduced only to shorten certain proofs.

Definition 3.2. A sequence $\left(V_{n}\right)$ of subsets of a topological space has property $\mathrm{B}$ if and only if $\bigcap\left\{\bar{V}_{n}: n \in N\right\}$ is nonempty and countably compact and $U$ open and $U \supset \bigcap\left\{\bar{V}_{n}: n \in N\right\}$ implies that some $V_{k} \subset U$.

Lemma 3.5. Suppose $X$ is a $T_{1}$-space and $\left(B_{n}\right)$ is a decreasing sequence of subsets of $X$ with property $\mathrm{B}$. Suppose $\left(A_{n}\right)$ is a decreasing sequence of nonempty subsets of $X$ such that for each $n$ some $A_{j} \subset B_{n}$. Then $\left(A_{n}\right)$ bas property $\mathrm{B}$. ( $A$ similar lemma is proved in [15].)

Lemma 3.6. Suppose $X$ is a completely regular $T_{0}$-space. Then $X$ is a $\mu$ space (complete $\mu$-space)(5) if and only if $X$ satisfies condition $\beta_{b}\left(\lambda_{b}\right)$.

(5) The part of Lemma 3.6 relating to complete $\mu$-space may be proved from Theorem 4 of [18] and Lemma 3.1. 
Proof. Let $\beta X$ denote the Stone-Čech compactification of $X$ and if $A \subset X$ $(A \subset \beta X)$ let $\bar{A}(\mathrm{cl}(A))$ denote the closure of $A$ in $X$ (in $\beta X)$. Suppose $X$ satisfies $\lambda_{b}$ or $\beta_{b}$. Then there exists a monotonically contracting sequence $\left(\biguplus_{n}\right)$ satisfying the appropriate condition of Definition 2.2 or 2.3 relative to $X$. By Lemma 3.3, there exists a primitive sequence $\left(\mathcal{H}_{n}\right)$ of $X$ in $X$ such that each $\mathcal{H}_{n} \subset \mathfrak{S}_{n}$. There exists a function $D$ on $\mathcal{H}_{1}$ such that if $U \in \mathcal{H}_{1}, D(U)$ is an open subset of $\beta X$ such that $D(U) \cap X=U$. Let $1 \theta_{1}$ denote $\left\{D(U): U \in \mathcal{H}_{1}\right\}$. Suppose $\left(\theta_{1}, \ldots, \theta_{n}\right.$ have been defined and are collections of open sets of $\beta X$ covering $X$ such that, for $k<n, x \in W \in\left(i_{k}\right.$ and $x \in X$ implies the existence of $W^{\prime} \in l_{k+1}$ such that $x \in W^{\prime} \subset W$. Let $W_{n+1}$ denote the collection of all sets of the form $V \cap U$ such that $U \in \mathbb{U}_{n}, V$ is open in $\beta X, V \cap X \in \mathcal{H}_{n+1}$, and $U$ contains a point of $V \cap X$ not in any predecessor of $V \cap X$ in $\mathcal{H}_{n+1}$. Then $\left(\mathbb{H}_{n}\right)$ is a monotonically contracting sequence of collections of open subsets of $\beta X$ covering $X$. For $\left(i_{1}\right.$ covers $X$. Suppose $\mathcal{H}_{n}$ covers $X$ and $x \in U \in \mathcal{U}_{n}$. There exist a first $H \in \mathcal{H}_{n+1}$ that contains $x$ and an open $V$ in $\beta X$ such that $V \cap X=H$. Therefore $x \in U \cap V \in \mathbb{l}_{n+1}$. Suppose $\left(W_{n}\right)$ is a decreasing representative of $\left(W_{n}\right)$. For each $n$, there exists $x \in$ $W_{n} \cap X$ such that the first element of $\mathcal{H}_{n}$ that contains $x$ includes $W_{n} \cap X$. Therefore there exists, by Lemma 3.4, a decreasing representative $\left(H_{n}\right)$ of ${ }^{n}\left(\mathcal{H}_{n}\right)$ such that, for each $n, H_{n}$ is the first element of $\mathcal{H}_{n}$ that includes a term of $\left(W_{n} \cap X\right)$. Suppose $\beta_{b}$ holds and there exists $x \in \bigcap\left\{W_{n}: n \in N\right\} \cap X$. Then $x \in \bigcap\left\{H_{n}: n \in N\right\}$. Since each $H_{n} \in \mathcal{G}_{n},\left(H_{n}\right)$ has property $\mathrm{B}$ (see Definition 3.2) and $\bigcap\left\{\bar{H}_{n}^{n}: n \in N\right\}$ is compact. Therefore, by Lemma 3.5, $\left(W_{n} \cap X\right)$ has property B. Similarly if $\lambda_{b}$ holds there is an $x \in \bigcap\left\{W_{n}: n \in N\right\} \cap X$ and $\left(W_{n} \cap X\right)$ has property B. Suppose in either case that $z \in \bigcap\left\{W_{n}: n \in N\right\}$ and $z \notin X$. Then $z \notin E=\bigcap\left\{\overline{W_{n} \cap X}: n \in N\right\}$. Since $E \subset \bigcap\left\{\bar{H}_{n}: n \in N\right\}$ it is compact. Hence there is a set $D$ open in $\beta X$ such that $E \subset D$ and $z \notin \mathrm{cl}(D)$. There exists $k$ such that $w_{k} \cap X \subset D \cap X$. Therefore $\operatorname{cl}\left(W_{k}\right)=\operatorname{cl}\left(W_{k} \cap X\right) C \operatorname{cl}(D \cap X)=\operatorname{cl}(D)$. But this implies $z \in \mathrm{cl}(D)$, which involves a contradiction. Hence $\bigcap\left\{W_{n}: n \in N\right\} \subset X$ so that $X$ is a $\mu$-space or a complete $\mu$-space accordingly as $\beta_{b}$ or $\lambda_{b}$ holds.

Now suppose $X$ is nonempty and either a $\mu$-space or a complete $\mu$-space. Then there exists a monotonically contracting sequence $\left(G_{n}^{\prime}\right)$ of open subsets of $\beta X$ covering $X$ and satisfying the condition of Definition 2.4 or 2.5. By Lemma 3.3 there exists a primitive sequence $\left(\mathcal{H}_{n}\right)$ of $X$ in $\beta X$ such that each $\mathcal{H}_{n}$ covers $X$ and is a subcollection of $\oint_{n}^{\prime}$. In [16] it is shown that under these conditions there exists a primitive sequence $\left(\mathbb{C}_{n}\right)$ of $X$ in $\beta X$ of collections of nonempty open subsets of $\beta X$ covering $X$ such that: (1) If $n<k$ and $x \in X$, then if $W$ and $W^{\prime}$ are the first elements of $W_{n}$ and $W_{k}$, respectively, that contain $x$ and $H$ is the first element of $\mathcal{H}_{n}$ that contains $x$, then $\mathrm{cl}\left(W^{\prime}\right) \subset W \cap H$. Let $\mathcal{C}_{1}$ denote $\left(\mathcal{C}_{1}\right)$. If $n>$ 1 , let $\mathcal{C}_{n}$ denote the collection of all sets of the form $U \cap W$ where $W \in(i){ }_{n}, U$ is open in $\beta X$ and contains a point of $W \cap X$ not in any predecessor of $W$ in ${ }^{n}{ }_{n}$, 
and, for some $W^{\prime} \in \mathcal{C}_{n-1}, \operatorname{cl}(U) \subset W^{\prime}$. For each $n$ let $\mathcal{S}_{n}=\left\{C \cap X: C \in \mathcal{C}_{n}\right\}$. Then each $\mathfrak{S}_{n}$ covers $X$. Suppose $x \in G \in \mathfrak{S}_{n}$. There exists $C \in \mathcal{C}_{n}$ such that $C \cap X=$ $G$. Let $W$ be the first element of $\mathcal{C}_{n+1}$ that contains $x$. There exists an open subset $U$ of $\beta X$ such that $x \in U$ and $\operatorname{cl}(U) \subset C$. Then $x \in U \cap W \in \mathcal{C}_{n+1}$ and $\operatorname{cl}(U \cap W) \subset C$. Hence $U \cap W \cap X \in \varrho_{n+1}$ and $\overline{U \cap W \cap X} \subset G$. Thus condition (1) of Lemmas 3.1 and 3.2 is satisfied. Suppose $\left(G_{n}\right)$ is a sequence such that $\bar{G}_{n+1}$ $\subset G_{n} \in \mathcal{G}_{n}$ for all $n$. Then by condition $(1)$ on $\left(\Theta_{n}\right)$ there exists a representative $\left(W_{n}\right)$ of $\left(\mathbb{Q}_{n}\right)$ such that, for each $n, W_{n}$ is the first element of $W_{n}$ that includes a term of $\left(G_{n}\right)$. With the use of an argument similar to the proof of Lemma 3.4 it follows that $\mathrm{cl}\left(W_{n+1}\right) \subset W_{n}$. By Lemma 3.4 there exists a decreasing representative $\left(H_{n}\right)$ of $\left(\mathcal{H}_{n}\right)$ such that, for each $n, H_{n}$ is the first element of $\mathcal{H}_{n}$ that includes a term of $\left(W_{n}\right)$. Suppose there exists $x \in \bigcap\left\{G_{n}: n \in N\right\}$. Then $\bigcap\left\{H_{n}: n \in N\right\} \subset X$ since $X$ is a $\mu$-space. Therefore $\bigcap\left\{W_{n}: n \in N\right\}$ is compact and has property B. Thus $\bigcap\left\{G_{n}: n \in N\right\}=\bigcap\left\{\bar{G}_{n}: n \in N\right\}=\bigcap\left\{\mathrm{cl}\left(G_{n}\right): n \in N\right\}$ is compact and has property $B$ by Lemma 3.5 and $X$ satisfies $\beta_{b}$ by Lemma 3.2. If $X$ is a complete $\mu$-space then $\bigcap\left\{H_{n}: n \in N\right\} \subset X$ and the argument just used together with the assumption that each $G_{n} \neq \varnothing$ shows that $\bigcap\left\{G_{n}: n \in N\right\}$ is compact and has property B. Thus $X$ satisfies $\lambda_{b}$ by Lemma 3.1.

Lemma 3.7. Suppose $f$ is a closed continuous mapping of a $T_{1}$-space $X$ onto a space $Y$. Suppose $y \in Y$ is such that for any open $U$ containing $y$ there is a sequence $\left(V_{n}\right)$ of open sets containing y such that any representative of $\left(V_{n}\right)$ bas a cluster point in $U$ and if $\mathcal{H}$ is a collection of open sets in $X$ covering $B=\operatorname{Fr}_{\mathrm{r}}\left(f^{-1}(y)\right)$ there exists a point-countable collection of open sets covering $B$ and refining $H$. Then every collection of open sets covering $B$ includes a finite subcollection $\mathcal{F}$ such that $(\bigcup \mathcal{F}) \cup f^{-1}(y)$ is open. If $X$ is also regular, then $B$ is compact.

Proof. Let $M$ denote $f^{-1}(y)$. If the conclusion is not satisfied there exists a point-countable collection $\mathcal{G}$ of open sets covering $B$ such that $\cup \mathcal{H} \cup M$ is not open for any finite $\mathfrak{H} \subset \mathcal{G}$. For each $x \in \bigcup \mathcal{G}$ let $\alpha(x)$ denote an infinite sequence such that $G$ is a term of $\alpha(x)$ if and only if $x \in G \in \biguplus$. Since $f$ is closed, there exists an open set $D$ in $X$ such that $D=f^{-1} f(D)$ and $M \subset D C$

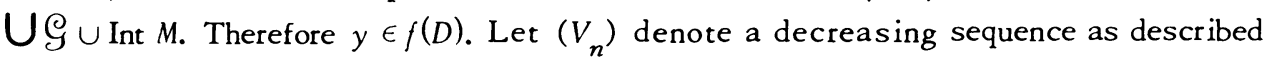
in the hypothesis relative to $y$ and $f(D)$ with $V_{1} \subset f(D)$. There exists a sequence $\left(x_{n}\right)$ such that $x_{1} \in\left(f^{-1}\left(V_{1}\right) \backslash M\right) \cap \bigcup \oint$ and, for each $n>1$,

$$
x_{n} \in\left[\left(f^{-1}\left(V_{n}\right) \backslash M\right) \cap \bigcup \mathcal{Y}\right] \mid \bigcup_{i=1}^{n-1} f^{-1}\left[\left\{f\left(x_{i}\right)\right\}\right]
$$

and, for each $n, x_{n+1}$ is not in any of the first $n$ terms of any of $\alpha\left(x_{1}\right), \ldots$, $a\left(x_{n}\right)$. Since $M$ is not open, there exists $x_{1} \in f^{-1}\left(V_{1}\right) \backslash M$. Assume $x_{1}, \cdots, x_{n}$ have been defined. Let $\mathcal{F}$ denote the collection of the first $n$ terms of each of 
$\alpha\left(x_{1}\right), \ldots, \alpha\left(x_{n}\right)$. Then $\cup \mathcal{F} \cup M$ is not open. There exists $G \in \mathcal{Y}$ such that

$$
A(G)=\left(f^{-1}\left(V_{n+1}\right) \cap G\right) \backslash \bigcup_{i=1}^{n} f^{-1}\left[\left\{f\left(x_{i}\right)\right\}\right] \not \subset \cup \mathcal{F} \cup M .
$$

For otherwise $B \subset \bigcup\{A(G): G \in \mathcal{G}\} \subset \cup \mathcal{F} \cup M$ which implies that $\bigcup \mathcal{F} \cup M$ is open. Select $x_{n+1} \in A(G) \backslash(\cup \mathcal{F} \cup M)$. The sequence $\left(f\left(x_{n}\right)\right)$ has a cluster point in $f(D)$. Since $f$ is closed, $\left(x_{n}\right)$ has a cluster point $w \in D$. Since $w \notin$ Int $M$, there

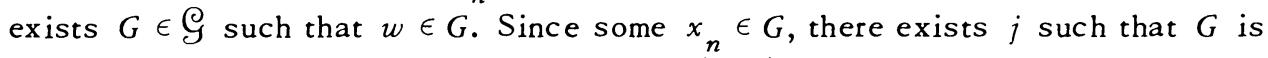
the $j$ th term of $\alpha\left(x_{n}\right)$. There exists $m>\max (n, j)$ such that $x_{m} \in G$. Since $x_{m}$ is not in the first $m-1$ terms of $\alpha\left(x_{n}\right)$ this involves a contradiction. It is straightforward to show that if $X$ is regular and $M$ is a closed subset of $X$ such that every collection of open sets covering $\operatorname{Fr}(M)$ includes a finite subcollection $\mathcal{F}$ such that $\bigcup \mathcal{F} \cup M$ is open, then $\operatorname{Fr}(M)$ is compact.

Lemma 3.8. Suppose $(X, \sigma)$ and $(Y, \tau)$ are topological spaces, $Y$ is $T_{1}$, and $f$ is a peripherally compact closed continuous mapping $X$ onto $Y$. Suppose that $\left(\mathcal{C}_{n}\right)$ is a primitive sequence of $X$ in $X$ such that each $\mathcal{C}_{n} \subset \sigma$.

Then there exists a sequence $\left(\mathcal{H}_{n}\right)$ of well-ordered subcollections of $\tau$ covering $Y$ and sequences $\left(M_{n}\right)$ and $\left(U_{n}\right)$ of functions such that for each $n \in N$ :

(P1) The domain of $M_{n}$ is $\mathcal{H}_{n}$ and its range is included in $Y$. For all $H \in \mathcal{H}_{n}$, $M_{n}(H)$ is a point of $H$ not in any predecessor of $H$. If $y \in Y$ and $\{y\}$ is open then $\{y\} \in \mathcal{H}_{n}$ and $M_{n}(\{y\})=y$.

(P2) If $y \in Y, j \in N, j<n$, and $H$ and $H^{\prime}$ are the first elements of $\mathcal{H}_{j}$ and $\mathcal{H}_{n}$, respectively, that contain $y$ then $H \supset H^{\prime}$. If $X$ is regular and $T_{0}, H \supset \bar{H}^{\prime}$.

(P3) The domain of $\mathcal{U}_{n}$ is the collection of nondegenerate elements of $\mathcal{H}_{n}$ and its range is included in the collection of all finite subcollections of $\tau$. If $H$ is in the domain of $\mathcal{U}_{n}, \mathcal{U}_{n}(H)$ covers both $\mathrm{Fr}_{\mathrm{r}}\left(f^{-1}\left(M_{n}(H)\right)\right)$ and $f^{-1}(H) \backslash f^{-1}\left(M_{n}(H)\right)$. Also each $U \in \mathcal{U}_{n}(H)$ contains $x \in \mathrm{Fr}\left(f^{-1}\left(M_{n}(H)\right)\right)$ such that if $k<n$ and $H^{\prime}$ is the first element of $\mathcal{H}_{k}$ that contains $M_{n}(H)$ then $U^{\prime} \in \mathcal{U}_{k}\left(H^{\prime}\right)$ and $x \in U^{\prime}$ implies $U \subset f^{-1}\left(H^{\prime}\right) \cap U^{\prime}$. If $X$ is regular and $T_{0}$ then $\bar{U} \subset f^{-1}\left(H^{\prime}\right) \cap U^{\prime}$. Furthermore, each $U \in \mathcal{U}_{n}(H)$ contains $z$ such that the first element of $\mathcal{C}_{n}$ that contains $z$ includes $U$.

Proof. It will be assumed that both $\sigma$ and the family $\Phi$ of all finite subcollections of $\sigma$ are well ordered. Let $<_{0}$ denote a well ordering of $Y$ such that if $y \in Y$ and $\{y\}$ is open then $y$ precedes (in the $<_{0}$ sense) every element of $Y$ which does not have the same property. Suppose there exist well-ordered collections $\mathcal{H}_{1}, \ldots, \mathcal{H}_{k}$, functions $M_{1}, \ldots, M_{k}$, and functions $\mathcal{U}_{1}, \ldots, \mathcal{U}_{k}$ satisfying (P1)-(P3) for all $n \leq k$ and let $<_{k-1}$ be a well ordering of $Y$. Define an ordering $<_{k}$ in $Y$ as follows: If $y, y^{\prime} \in Y$ and $H$ and $H^{\prime}$ are the first elements of $\mathcal{H}_{k}$ that contain $y$ and $y^{\prime}$ respectively, then $y<_{k} y^{\prime}$ if and only if either (1) $H$ precedes 
$H^{\prime}$ in $\mathcal{H}_{k}$ or (2) $H=H^{\prime}$ and $y<_{k-1} y^{\prime}$. It may be seen that $\leq_{k}$ well orders $Y$. Let $Y_{k}$ denote $Y$ under this ordering. Now a sequence function $g$ of type $Y_{k}$ in $\tau$ is to be defined [5]. For $y \in Y_{k}$ let $s(y)=\left\{z \in Y_{k}: z<_{k} y\right\}$. Suppose $y \in Y_{k}$ and $t$ : $s(y) \rightarrow \tau$. If $\{y\}$ is open, let $g(t)=\{y\}$. If $\{y\}$ is not open, consider two cases. Case (i). There exists $z \in s(y)$ such that $y \in t(z)$. Let $y^{\prime}$ denote the first such $z$ in $Y_{k}$ and let $g(t)=t\left(y^{\prime}\right)$. Case (ii). If $z<_{k} y$ then $y \notin t(z)$. Let $H_{j}$ denote the first element of $\mathcal{H}_{j}$ that contains $y$. Let $M$ denote $\operatorname{Fr}\left(f^{-1}(y)\right)$ and suppose $x \in M$. Let $W$ denote the intersection of all sets of $\mathcal{U}_{j}\left(H_{j}\right)$ that contain $x$ for $1 \leq j \leq k$ and let $V$ denote the first element of $C_{k_{+}+1}$ that contains $x$. There exists a first $U_{x}$ in $\sigma$ such that $x \in U_{x} \subset W \cap V \cap f^{-1}\left(H_{k}\right)$. If $X$ is regular and $T_{0}$, it may also be required that $\bar{U}_{x} \subset W \cap V \cap f^{-1}\left(H_{k}\right)$. Since $\operatorname{Fr}\left(f^{-1}(y)\right)$ is compact, there exists a first element $\mathcal{U}$ of $\Phi$ that is a subcollection of $\left\{U_{x}: x \in M\right\}$ covering $M$. Define $g(t)$ as $\left\{z \in Y: f^{-1}(z) \subset \cup \cup \cup\right.$ Int $\left.f^{-1}(y)\right\}$. Since $f$ is closed, $g(t) \in \tau$. By the transfinite recursion theorem there exists a unique function $U: Y_{k} \rightarrow \tau$ such that $U(y)=g(U \mid s(y))$ for all $y \in Y_{k}$.

For every $H$ in the range of $U$, let $M_{k+1}(H)$ denote the first element of $Y_{k}$ such that $U\left(M_{k+1}(H)\right)=H$. Then $M_{k+1}$ is a one-to-one function on the range of $U$ into $Y_{k}$. This permits the definition of a well ordering $\preceq$ by $H \prec H^{\prime}$ if and only if $M_{k+1}(H)<_{k} M_{k+1}\left(H^{\prime}\right)$ for $H, H^{\prime}$ in the range of $U$. Call the resulting well-ordered set $\mathfrak{H}_{k+1}$.

1. If $y<{ }_{k} M_{k+1}(H)$ then $M_{k+1}(H) \notin U(y)$. For if $M_{k+1}(H) \in U(y)$ there exists a first $z \in s\left(M_{k+1}(H)\right)$ such that $M_{k+1}(H) \in U(z)$. Thus $H=U\left(M_{k+1}(H)\right)=$ $g\left(U \mid s\left(M_{k+1}(H)\right)\right)=U \mid s\left(M_{k+1}(H)\right)(z)=U(z)$ contrary to the definition of $M_{k+1}(H)$.

2. If $y \in Y_{k}$ the first element $H$ of $\mathcal{H}_{k+1}$ that contains $y$ is $U(y)$. For $y \in$ $U(y)$ since $g(U \mid s(y))=U(y)$ contains $y$. If $H \prec U(y)$ and $y \in H$ then $y \in$ $U\left(M_{k+1}(H)\right)$ where $M_{k+1}(H)<_{k} y$. For $M_{k+1}(H) \leq_{k} y$ and $y \neq M_{k+1}(H)$ since $H \neq$ $U(y)$. By definition of $g$, Case (i), $U(y)=U(z)$ for the first $z<_{k} y$ such that $y \in$ $U(z)$. From the definition of $z, z=M_{k+1}(U(z)) \leq_{k} M_{k+1}(H)$. Thus $U(y)=U(z) \preceq H$ $\prec U(y)$ which is a contradiction.

Suppose $y \in Y_{k}$ and $\{y\}$ is open. Then $U(y)=\{y\}$ and $M_{k+1}(\{y\})=y$. From this and 2 it follows that $(\mathrm{P} 1)$ is satisfied. To show that (P2) is satisfied suppose $y \in Y$ and $H$ and $H^{\prime}$ are the first elements of $\mathcal{H}_{k}$ and $\mathcal{H}_{k+1}$ respectively that contain $y$. Let $G$ be the first element of $\mathcal{H}_{k}$ that contains $M_{k+1}\left(H^{\prime}\right)$. Since $H^{\prime}=$ $U\left(M_{k+1}\left(H^{\prime}\right)\right)=g\left(U \mid s\left(M_{k+1}\left(H^{\prime}\right)\right)\right)$ it follows from the definition of $g$ that $G \supset H^{\prime}$ (if $X$ is regular and $T_{0}, G \supset \overrightarrow{H^{\prime}}$ ). Therefore $y \in G$ so that $H \preceq G$. If $H \prec G$, then $y<_{k}$ $M_{k+1}\left(H^{\prime}\right)$. Since 2 implies that $U(y)=H^{\prime}$, this is impossible. Therefore $H=G$.

To show that (P3) holds, consider any nondegenerate element $H$ of $\mathcal{H}_{k+1}$. There exists, by the definition of $g$, a first $U \in \Phi$ such that $H=\left\{z \in Y: f^{-1}(z) \subset\right.$ $\cup \mathcal{U} \cup$ Int $\left.f^{-1}\left(M_{k+1}(H)\right)\right\}$. Let $\mathcal{U}_{k+1}(H)$ denote $\mathcal{U}$. Then $\mathcal{U}_{k+1}(H)$ is finite and covers $\operatorname{Fr}\left(f^{-1}\left(M_{k+1}(H)\right)\right)$ and $f^{-1}(H) \backslash f^{-1}\left(M_{k+1}(H)\right)$. If $U \in \mathcal{U}_{k+1}(H)$ then $U=U_{x}$ 
for some $x \in \mathrm{Fr}_{\mathrm{r}}\left(f^{-1}\left(M_{k+1}(H)\right)\right)$. If $j<k+1$ and $H^{\prime}$ is the first element of $\mathcal{H}_{j}$ that contains $M_{k+1}(H)$ and $U^{\prime} \in \mathcal{U}_{j}\left(H^{\prime}\right)$ contains $x$ then the definition of $g$, Case (ii), shows that $U_{x}=U \subset U^{\prime} \cap V \cap f^{-1}\left(H^{\prime}\right)$ where $V$ is the first element of $\mathcal{C}_{k+1}$ that contains $x$. In the regular $T_{0}$ case $\bar{U} \subset U^{\prime} \cap V \cap f^{-1}\left(H^{\prime}\right)$. It follows that (P3) is satisfied. From the definition of $g$ it should be clear how to obtain $\mathcal{H}_{1}, M_{1}$ and $\mathcal{U}_{1}$ such that the applicable parts of $(\mathrm{P} 1)-(\mathrm{P} 3)$ hold. Thus, by mathematical induction, the sequences exist.

\section{Proofs of theorems.}

Proof of Theorem 2.1. Since the space $X$ is assumed to be regular and $T_{0}$ the same is true of $Y$. Thus it suffices to show that $Y$ has a monotonically contracting sequence satisfying the appropriate conditions of Lemmas 3.1 and 3.2 whenever $X$ has one. Suppose $X$ has a sequence $\left(G_{n}\right)$ satisfying the appropriate conditions of Lemmas 3.1 or 3.2 accordingly as $X$ satisfies $\lambda_{b}, \lambda_{c}, \beta_{b}$ or $\beta_{c}$. There exists a primitive sequence $\left(\mathcal{C}_{n}\right)$ of $X$ in $X$ such that each $\mathcal{C}_{n} \subset \mathcal{S}_{n}$ by Lemma 3.3. By Lemma 3.8 there exists a sequence $\left(\mathcal{H}_{n}\right)$ with properties (P1)-(P3) relative to $f$ and $\left(\mathcal{C}_{n}\right)$. Let $W_{1}$ denote $\mathcal{H}_{1}$. If $W_{1}, \ldots, W_{n}$ have been def ined let $i_{n+1}$ denote the collection of all sets of the form $U \cap H$ where $H \in \mathcal{H}_{n+1}, U$ is open, $U$ contains a point of $H$ not in any predecessor of $H$ in $\mathcal{H}_{n+1}$, and for some $H^{\prime} \in \mathcal{H}_{n}, \bar{U} \subset H^{\prime}$. By an argument used in the proof of Lemma 3.6 it may be seen that each $(i)_{n}$ covers $Y$ and if $y \in W \in(i)_{n}$ there exists $\left.W^{\prime} \in 7_{n+1}\right)_{n}$ such that $y \in W^{\prime}$ and $\overline{W^{\prime}} \subset W$. It is assumed that $X \neq \varnothing$ and that no element of $\stackrel{H}{H}_{1}^{+1}$ is empty. It follows that all elements of each $t_{n}$ are nonempty.

I. Suppose $\left(W_{n}\right)$ is a representative of $\left.\left({ }^{0}\right)_{n}\right)$ such that each $\bar{W}_{n+1} \subset W_{n}$. For each $n$ there exists a first $H_{n} \in \mathcal{H}_{n}$ that includes a term of $\left(W_{n}\right)$. By Lemma 3.4, $\left(H_{n}\right)$ is decreasing. Suppose $n<k$. If $H$ is the first element of $\mathcal{H}_{n}$ that contains $M_{k}\left(H_{k}\right)$ then $H \supset \bar{H}_{k}$. Therefore $H$ does not precede $H_{n}$. Since $H_{n}^{n} \supset H_{k}$ it follows that $H=H_{n}$ and $H_{n} \supset \bar{H}_{k}$. If some $W_{n}=\{y\}$ for $y \in Y$ then $\bigcap\left\{W_{n}: n \in N\right\}=\{y\}$ and $\left\{W_{n}: n \in N\right\}$ is a base at $\{y\}$. Thus it may be assumed that no $W_{n}$ is of this form.

II. Suppose $\beta_{c}$ holds and there exists $y \in \bigcap\left\{W_{n}: n \in N\right\}$. Then $y \in J=$ $\bigcap\left\{H_{n}: n \in N\right\}$. Let $C_{n}$ denote $f^{-1}\left(M_{n}\left(H_{n}\right)\right)$ and $\mathcal{U}_{n}$ denote $\mathcal{U}_{n}\left(H_{n}\right)$. Suppose $x \in \operatorname{Fr}\left(f^{-1}(y)\right)$. With the use of (P3) and König's lemma [8] or [11, p. 47] it may be seen that there exists a sequence $\left(U_{n}\right)$ such that, for all $n, \bar{U}_{n+1} \subset U_{n} \cap f^{-1}\left(H_{n}\right)$ and $x \in U_{n} \in$ $\mathrm{U}_{n}$. By (P3) for each $n$ there is a first $A_{n} \in \mathcal{C}_{n}^{n+1}$ that includes a term of $\left(U_{n}\right)$ and by Lemma 3.4, $\left(A_{n}\right)$ is decreasing. It follows from Lemma 3.5 and the fact that each $\mathcal{C}_{n} \subset \mathcal{G}_{n}$ that $\left(U_{n}\right)$ has property B. For each $n$ there exists $x_{n} \in U_{n} \cap C_{n}$. The sequence $\left(x_{n}\right)$ has a cluster point $u \in \bigcap\left\{U_{n}: n \in N\right\}$. Therefore $f(u) \in$ $\bigcap\left\{f\left(U_{n}\right): n \in N\right\} \subset J$. Let $C$ denote $f^{-1}(f(u))$. Suppose $W$ is open and $W \supset J$. Then $C \subset f^{-1}(W)$. For each $z \in \operatorname{Fr}(C)$, with the use of König's lemma, (P3), and 
a representative of $\left(\mathcal{C}_{n}\right)$ as above, there exists a sequence $\left(V_{n}\right)$ with property $\mathrm{B}$ such that each $V_{n} \in \mathbb{U}_{n}^{n}, \bar{V}_{n+1} \subset V_{n} \subset f^{-1}\left(H_{n}\right)$, and $z \in \bigcap\left\{V_{n}: n \in N\right\} \subset f^{-1}(W)$. Then for each $z \in \mathrm{Fr}_{\mathrm{r}}(C)$ there exists $j$ and $U \in \mathcal{U}_{j}$ such that $z \in U \subset f^{-1}(W)$. Since $\operatorname{Fr}(C)$ is compact there exist $k$ and a subcollection $\mathfrak{D}$ of $\mathcal{U}_{1} \cup \ldots \cup \mathcal{U}_{k}$ such that $\operatorname{Fr}(C) \subset \bigcup \mathfrak{D} \subset f^{-1}(W)$. Let $H$ denote $\left\{y \in Y: f^{-1}(y) \subset \bigcup \mathfrak{D} \cup \operatorname{Int}(C)\right\}$. Then $H$ is open and $u \in f^{-1}(H)$. Therefore, for some $j>k, x_{j} \in f^{-1}(H)$ and thus $C_{j} \subset \cup \mathfrak{D} \cup \operatorname{Int}(C)$. If $U \in \mathcal{U}_{j}, U$ contains $x \in \operatorname{Fr}\left(C_{j}\right)$ such that if $n<j$ any element of $\mathcal{U}_{n}$ which contains $x$ includes $U$. Thus $\cup U_{j} \subset \cup \mathfrak{D}$ and $f^{-1}\left(H_{j}\right) \subset$ $\cup \mathcal{U}_{j} \cup C_{j} \subset f^{-1}(W)$. It follows that any open set which includes $J$ includes some $H_{k}$. Suppose $M$ is an infinite subset of $J$. If $M$ includes an infinite subset of $\left\{M_{n}\left(H_{n}\right): n \in N\right\}$ then $M$ has a limit point as follows from the fact that $\left(x_{n}\right)$ has a cluster point. If $M$ contains no such subset then, for each $n, f^{-1}(M)$ includes an infinite subset of $f^{-1}\left(H_{n}\right) \backslash\left\{C_{k}: k \in N\right\}$. Therefore, for each $n$, the set $\mathcal{U}_{n}^{\prime}=\{U \epsilon$ $\left.U_{n}:|f(U) \cap M| \geq \boldsymbol{N}_{0}\right\}$ is not empty. By König's lemma there exists a sequence $\left(U_{n}\right)$ such that each $\bar{U}_{n+1} \subset U_{n} \in \mathcal{U}_{n}^{\prime}$. It follows as above that $\bigcap\left\{U_{n}: n \in N\right\}$ has property B. There exists a sequence $\left(y_{n}\right)$ of distinct points such that each $y_{n} \in M \cap$ $f\left(U_{n}\right)$. If $y_{n}=f\left(z_{n}\right)$, where $z_{n} \in U_{n}$, there is a limit point $z$ of $\left\{z_{n}: n \in N\right\}$ belonging to $\bigcap\left\{U_{n}: n \in N\right\}$. Therefore $f(z)$ is a limit point of $M$, so that $J$ is countably compact and thus has property B. It follows from Lemma 3.5 that $\bigcap\left\{W_{n}: n \in N\right\}$ is countably compact and has property $\mathrm{B}$. Therefore $Y$ satisfies $\beta_{c}$ if $X$ does. Suppose $X$ satisfies $\beta_{b}$ and $\mathbb{Q}$ is a collection of open sets covering $J$. If $x \in$ $f^{-1}(J) \backslash\left\{C_{k}: k \in N\right\}$ there exists a sequence $\left(U_{n}\right)$ such that $x \in U_{n+1} \subset \bar{U}_{n+1} \subset U_{n}$ and $U_{n} \in \mathcal{U}_{n}$ for all $n$. Each $\bigcap\left\{U_{n}: n \in N\right\}$ is a compact subset of $f^{-1}(J)$. Thus $f^{-1}(J) \backslash\left\{C_{k}: k \in N\right\}$ is covered by countably many compact subsets of $f^{-1}(J)$. It follows that $f^{-1}(J)$ is covered by countably many elements of $f^{-1}(\mathcal{C})$. Therefore $J$ has the Lindelöf property and, since it is $T_{1}$ and countably compact, it is compact. Thus $\bigcap\left\{W_{n}: n \in N\right\}$ is compact so $Y$ satisfies $\beta_{b}$.

III. If $\lambda_{c}\left(\lambda_{b}\right)$ holds, then, using part of the argument of II, there exists $y \epsilon$ $\bigcap\left\{W_{n}: n \in N\right\}$. Then the argument of II shows that $\bigcap\left\{W_{n}: n \in N\right\}$ has property $\mathrm{B}$ (and is compact in case $\left.\lambda_{b}\right)$, and therefore $Y$ satisfies $\lambda_{c}\left(\lambda_{b}\right)$.

Proof of Theorem 2.3. If $X$ is a completely regular $T_{0} \mu$-space it satisfies $\beta_{b}$ by Lemma 3.6. Therefore $Y$ satisfies $\beta_{b}$ by Theorem 2.1. Since $Y$ is completely regular and $T_{0}$ it is a $\mu$-space by Lemma 3.6. A similar argument proves the second part of the theorem.

Proof of Theorems 2.2 and 2.4. Under the hypothesis on $f, X$, and $Y$ it follows from Lemma 3.7 that $f$ is peripherally compact. Thus the results follow from Theorems 2.1 and 2.3.

Proof of Theorems 1.1 and 1.2. These theorems are corollaries to the propositions of I and II of the introduction and Theorem 2.1. 


\section{REFERENCES}

1. A. V. Arhangel'skir, On a class of spaces containing all metric and all locally compact spaces, Mat. Sb. 67 (109) (1965), 55-88; English transl., Amer. Math. Soc. Transl. (2) 92 (1970), 1-39. MR 32 \#8299; MR $42 \# 3$.

2. V. V.Filippov, On the perfect image of a paracompact p-space, Dokl. Akad. Nauk SSSR 176 (1967), 533-535 = Soviet Math。Dokl。8 (1967), 1151-1153. MR 36 \#5903.

3. M. Fréchet, Sur quelques points du calcul fonctionnel, Rend. Circ. Mat. Palermo 22 (1906), 1-74.

4. Z. Frolik, On the topological product of paracompact spaces, Bull. Acad. Polon. Sci. Sér. Sci. Math. Astronom. Phys。 8 (1960), 747-750. MR 23 \#A2859.

5. P. R. Halmos, Naive set theory, University Ser. in Undergraduate Math., Van Nostrand, Princeton, N. J., 1960. MR $22 \# 5575$.

6. T. Ishii, On closed mappings and M-spaces. II., Proc. Japan Acad. 43 (1967), 757761. MR $36 \# 5904$.

7. J. L. Kelley, General topology, Van Nostrand, Princeton, N. J., 1955. MR 16, 1136.

8. D. König, Sur les correspondences multivoques, Fund. Math.8 (1926), 114-134.

9. E. Michael, Another note on paracompact spaces, Proc. Amer. Math. Soc. 8 (1957), 822-828. MR 19, 299.

10. - A note on closed maps and compact sets, Israel J. Math. 2 (1964), 173-176. MR 31 \#1659.

11. R. L. Moore, Foundations of point set theory, rev.ed., Amer. Math. Soc. Colloq. Publ., vol. 13, Amer. Math. Soc., Providence, R. I., 1962. MR 27 \#709.

12. K. Morita, Products of normal spaces with metric spaces, Math. Ann. 154 (1964), 365-382. MR 29 \#2773.

13. - Some properties of M-spaces, Proc. Japan Acad. 43 (1967), 869-872. MR $37 \# 3517$.

14. H. H. Wicke, The regular open continuous images of complete metric spaces, $\mathrm{Pa-}$ cific J. Math. 23 (1967), 621-625. MR $36 \# 2118$.

15. - Open continuous images of certain kinds of $M$-spaces and completeness of mappings and spaces, General Topology and Appl. 1 (1971), 85-100. MR 43 \#8060.

16. H. H. Wicke and J. M. Worrell, Jr., On topological completeness of first countable Hausdorff spaces. I, Fund. Math. 75 (1972), 209-222.

17. - On a class of spaces containing Arhangel'skil̆'s p-spaces, Notices Amer. Math. Soc. 14 (1967), 687. Abstract \#648-188.

18. - On the open continuous images of paracompact $\check{C}$ ech complete spaces, Pacific J. Math. 37 (1971), 265-275.

19. J. M. Worrell, Jr. and H. H. Wicke, Characterizations of developable topological spaces, Canad. J. Math. 17 (1965), 820-830. MR 32 \#427.

20. J. M. Worrell, Jr., Upper semicontinuous decompositions of developable spaces, Proc. Amer. Math. Soc. 16 (1965), 485-490. MR 31 \#6207.

21. Upper semicontinuous decompositions of spaces having bases of countable order, Portugal. Math. 26 (1967), 493-504. MR $41 \# 2626$.

22. - A perfect mapping not preserving the p-space property, presented at Pittsburgh Conference on General Topology 1970.

SANDIA LABORATORIES. ALBUQUERQUE, NEW MEXICO 87115

DEPARTMENT OF MATHEMATICS, OHIO UNIVERSITY, ATHENS, OHIO 45701 (Current address of both authors) 